\title{
Apuntes críticos al documento Marco conceptual para la preparación y presentación de los estados financieros*
}

\section{Documento aprobado por el Consejo del IASC y adoptado por el IASB en abril de $2001^{* *}$}

Carlos Arturo Gómez Restrepo***

Gómez R., C. A. (2010). Apuntes críticos al documento Marco conceptual para la preparación y presentación de los estados financieros. Documento aprobado por el Consejo del IASC y adoptado por el IASB en abril de 2001. Revista Activos, 15, 123-133.

JEL: F02, M41

Recibido: 4 de octubre de 2010 Aprobado: 22 de noviembre de 2010

\section{Resumen}

El IASC presentó en 1989 el documento denominado Marco conceptual, que establece conceptos que subyacen en la preparación y presentación de estados financieros para usuarios externos. El presente artículo tiene como objetivo formular algunas reflexiones críticas

* Artículo de interés académico y profesional

** $\quad$ El autor agradece los comentarios y sugerencias recibidas de los dos evaluadores secretos.

*** Decano Facultad de Contaduría Pública Universidad Santo Tomás. Profesor de la Facultad de Ciencias Económicas de la Universidad Nacional de Colombia. 
sobre él, buscando servir de pretexto para la discusión académica del mismo, a propósito del actual debate sobre la aplicación de las normas internacionales de contabilidad, y en el caso particular colombiano, sobre el cumplimiento de la Ley 1314 de 2009. Para esto, analiza aspectos positivos como introducción de nuevos modelos, y demuestra sus deficiencias, como las limitaciones en su aplicabilidad y el tratamiento limitado y ambiguo, que no logra dejar sentadas unas definiciones conceptuales, típicas de un marco conceptual.

\title{
Palabras clave
}

Marco conceptual, normas internacionales de contabilidad, activos, pasivos, patrimonio

Gómez R., C. A. (2010). Critical notes on the document "Framework for the preparation and presentation of financial statements." Document approved by the IASC Board and adopted by the IASB in April 2001. Activos Review, 15, 123-133.

\begin{abstract}
The IASC introduced in 1989 the document called Framework, which provides concepts underlying the preparation and presentation of financial statements for external users. This article aims to make some critical reflections on it, looking for an excuse, for the academic discussion of it in regards to the current debate on the application of international accounting standards, and in the case of Colombia, on compliance of Law 1314 of 2009. For this reason, it analyses positive aspects such as the introduction of new models, and shows its shortcomings and limitations in its applicability and the limited and ambiguous treatment that fails to lay down conceptual definitions, typical of a conceptual framework.
\end{abstract}

\section{Keywords}

Framework, international accounting standards, assets, liabilities, equity 
Gómez R., C. A. (2010). Notes critiques sur le document "Cadre conceptuel pour la préparation et la présntation des états financiers". Document approuvé par le Conseil de l' IASC et adopté par l' IASB en avril 2001. Revue Activos, 15, 123-133.

\section{Résumé}

En 1989, l'IASC a présenté le document intitulé Cadre Conceptuel, qui établit des concepts sous-jacents dans la préparation et présentation détats financiers pour des usagers externes. Le présent article a comme objectif de formuler quelques réflexions critiques sur le cadre, servant de prétexte à sa discussion académique à propos du débat actuel sur l'application des normes internationales de comptabilité et, dans le cas particulier colombien, sur l'exécution de la loi 1314 de 2009. Dans ce but, il en analyse certains aspects positifs tels que l'introduction de nouveaux modèles et en démontre des déficiences, comme les limitations dans son applicabilité et son traitement limité et ambigu qui ne permet pas d'établir clairement la définition de certains concepts, comme on l'attend d'un cadre conceptuel.

\section{Mots-clé}

Cadre conceptuel, normes internationales de comptabilité, actifs, passifs, patrimoine

\section{Introducción}

Según el IASC ${ }^{1}$, las normativas contables de los países son diferentes porque se elaboran de acuerdo con sus propias circunstancias económicas y legales, y porque pretenden atender las necesidades de distintos grupos de usuarios, lo que de alguna manera ocasiona que los estados financieros, aunque similares, presenten diferencias. Para cumplir con su misión de reducirlas, la entidad cree que es más efectivo centrarse en los estados financieros que suministran información sobre la que los agentes toman decisiones económicas. Con esta perspectiva, el IASC presentó en 1989 el documento denominado Marco conceptual, que establece conceptos que subyacen en la preparación y presentación de estados financieros para usuarios externos.

Un aspecto positivo del Marco conceptual es que busca introducir nuevos modelos que, considera, pueden ser útiles para suministrar información adecuada para la toma de decisiones económicas. Estos modelos son adicionales al modelo contable basado en el costo 
histórico y/o en el concepto de mantenimiento de capital financiero en términos nominales, que ha imperado por muchas décadas como sustento teórico de la disciplina contable.

Sin embargo, el documento del IASC presenta deficiencias que se pueden resumir en dos puntos: 1) Por ser un marco conceptual, su alcance debería ser de aplicación general, sin presentar limitaciones en cuanto a su aplicabilidad a estados financieros para propósitos especiales, y sin quedar supeditado a las normas internacionales de contabilidad.2) Aunque una parte importante de su contenido presenta una proposición que trata de exponer de manera precisa y única la comprensión de un término, en este caso contable, logrando tipificaciones y diferenciaciones que en la mayoría de los casos se pueden considerar teoréticas, introduce algunos elementos sustantivos en la discusión teórica, con un tratamiento bastante limitado y ambiguo, que aunque deja percibir una problemática por resolver, no logra dejar sentadas las definiciones conceptuales típicas de un marco conceptual.

El presente documento tiene como objetivo formular algunas reflexiones críticas sobre el Marco conceptual, buscando servir de pretexto para la discusión académica del mismo, a propósito del actual debate sobre la aplicación de las normas internacionales de contabilidad y, en el caso particular colombiano, sobre el cumplimiento de la Ley 1314 de 2009.

\section{Los aspectos positivos}

El tratamiento que el Marco conceptual da al patrimonio inicia con una definición elemental, según la cual es la parte residual de los activos de la entidad una vez deducidos todos sus pasivos. Más adelante complementa esta definición, indicando que su valor depende de la valoración que se haya efectuado de los activos y de los pasivos. Pero más importante aún, deja planteados dos elementos consustanciales en la discusión sobre el patrimonio: primero, la diferencia que existe entre su valor en libros y en el mercado de valores, y segundo, la diferencia del patrimonio con respecto al valor de venta de cada uno de los activos, $o$ al valor de venta de todo el negocio en marcha.

La primera diferencia constituye un tema fundamental para la contabilidad, toda vez que plantea el interrogante sobre qué tipo de valoraciones debe expresar si se tiene en cuenta que las decisiones económicas de los agentes, a las cuales quiere servir la contabilidad, por lo general se toman sobre la base de valores de mercado. Esta diferencia ha sido expresada en Colombia mediante el indicador $q$ de Tobin, que refleja la relación entre el precio de 
mercado de una acción y su valor intrínseco². Sin embargo, la $q$ de Tobin (Tobin, 1969) tiene un significado mucho más profundo, ya que relaciona los precios de mercado con la productividad física de los activos: es diferente de uno, si aquellos precios no reflejan completamente la productividad física del activo.

Hay quienes atribuyen esa diferencia al capital intelectual. Según ellos, la diferencia se presenta por el conocimiento implícito y explícito ${ }^{3}$ que tienen los integrantes de una organización y, que en últimas, determina la capacidad de la empresa para generar flujos de caja futuros, independientemente del valor de sus activos y pasivos ${ }^{4}$.

La segunda diferencia reconoce la dicotomía entre el valor contable del patrimonio y el valor de venta de sus activos, de una parte, y el valor de venta del negocio, por la otra. Este reconocimiento explicita por lo menos dos problemas: el primero nos plantea de nuevo que la valoración a precios de mercado difiere de la valoración contable. Las decisiones de los agentes se basan en precios del mercado, por lo que terminan utilizando la información contable de manera marginal.Y segundo, el valor de venta de un negocio se calcula utilizando varios métodos de valoración, dentro de los cuales sobresale el de flujos de caja libres descontados ${ }^{5}$. Hicks (1939) se pregunta: ¿cuál será el plan de producción preferido por un empresario? En un análisis estático, será el que haga que el empresario lleve al máximo su excedente de sus ingresos sobre sus costos. Pero cuando utilizamos un análisis dinámico, se hace evidente que el empresario puede esperar no sólo un excedente sino una corriente de excedentes que se repitan en el futuro. Una manera fundamental de presentar el análisis dinámico es considerarlo en términos de un valor capitalizado de la corriente de excedentes. Ahora bien, cabe preguntarse si el valor de un negocio corresponde a los flujos de caja futuros descontados, ¿qué relevancia puede tener el valor patrimonial que presenta la contabilidad?

Las dos diferencias señaladas, aunque importantes porque invitan a un análisis sobre una problemática específica, cual es la de establecer metodologías de valoración, de activos, de

2 El valor intrínseco de una acción se calcula dividiendo el valor del patrimonio entre el número de acciones en circulación.

3 Sobre conocimiento implícito y explícito puede consultarse: Michael Oakeshott (1991), que distinguía entre "conocimiento práctico" y "conocimiento científico"; Hayek (1972) diferenciaba los conceptos de "conocimiento disperso" y "conocimiento centralizado"; Michael Polanyi (1959), quien diferenciaba "conocimiento tácito" de "conocimiento articulado", y Mises (1995), que separaba el "conocimiento sobre eventos únicos" del "conocimiento sobre el comportamiento de toda una clase de fenómenos".

4 Véase Edvinsson y Malone (1998).

5 Puede consultarse Copeland, T. (2000). Valuation: Measuring and Managing the Value of Companies, 3rd Edition. McKinsey \& Company Inc. 
pasivos y por tanto de patrimonio, no pasan de ser una ilustración sencilla, que por tratarse de un marco conceptual deberían trascender el planteamiento de problemas y convertirse en un marco de referencia conceptual que incluya definiciones y conceptos precisos para el avance del conocimiento contable.

De otra parte, el Marco conceptual propone dos conceptos de capital: el financiero, que considera el dinero invertido: el capital es sinónimo de activos netos o patrimonio neto.Y el concepto físico (capacidad productiva): capital es la capacidad operativa. Estos dos conceptos son flexibles de acuerdo con los usuarios de la información, y llevan a la definición de mantenimiento de capital. Mantenimiento del capital financiero, según el cual se obtiene ganancia si el importe financiero de los activos netos al final del periodo excede al importe financiero de los activos netos en el inicio del mismo; y mantenimiento del capital físico, según el cual se obtiene ganancia sólo si la capacidad productiva en términos físicos al final del periodo excede la capacidad productiva en términos físicos en el comienzo del mismo.

La definición de mantenimiento del capital propuesto por el Marco conceptual es coincidente con Knight (1921, p. 16), quien propone que el beneficio debe ser computado en relación con cierto intervalo de tiempo a fecha fija, es decir, un periodo contable de longitud arbitraria. El beneficio de una unidad económica se calcula como la diferencia entre ingresos y gastos en cuenta corriente (con una contabilidad racional) durante el periodo establecido, aumentado o disminuido por una variación del total activo en el mismo periodo.

Tanto el concepto de capital como el de mantenimiento de capital proporcionan el punto de referencia para medir la ganancia y permiten distinguir entre rendimiento sobre el capital y recuperación del capital. Sólo las entradas de activos que excedan las cantidades necesarias para mantener el capital pueden considerarse como ganancia, y por tanto como rendimiento del capital.

Finalmente, introduce la noción de poder adquisitivo constante cuando plantea que sólo la parte del incremento en los precios de los activos que exceda en incremento el nivel general de precios se considera como resultados. Nuevamente se coincide con Knight (1921), que argumenta que para la medición del beneficio de una unidad económica es ideal suponer siempre una unidad monetaria de valor constante.

El Marco conceptual, aunque se limita a insinuar algunos núcleos de problematización del patrimonio, reconoce las limitaciones de la valoración de activos, pasivos, del patrimonio y de la utilidad en la contabilidad, e invita al lector a pensar en la solución de problemas 
reales de la disciplina contable, sin proponer una conceptualización que oriente hacia ella, como debería hacerlo de acuerdo con su propia naturaleza.

\section{Las deficiencias}

Como se presentó en la introducción del presente documento, a continuación se explicitan las dos deficiencias enunciadas.

Primera deficiencia: "Por ser un marco conceptual, su alcance debería ser de aplicación general, sin presentar limitaciones en cuanto a su aplicabilidad a estados financieros para propósitos especiales y sin quedar supeditado a las normas internacionales de contabilidad".

Un marco conceptual debe incluir la definición de términos básicos. El conocimiento científico es conceptual y está construido a través de conceptos que se aglutinan en sistemas interrelacionados de múltiples y diversas maneras. Un concepto es una abstracción de la realidad; su fin es simplificar resumiendo un grupo de observaciones que se pueden clasificar bajo un mismo nombre. Para aprehender las ciencias es necesario el dominio de los conceptos que conforman el lenguaje de cada ciencia (Borsotti, 1990).

Las definiciones de términos básicos, los conceptos, que constituyen el propio entramado del Marco conceptual, presentado por el IASC, deben constituirse en el vehículo para acceder al conocimiento contable. La aprehensión de las ciencias no puede limitarse a unos determinados y limitados fines prácticos, debe ser general. El Marco conceptual debe aplicarse a todos los estados financieros para no perder su razón de ser.

A través del Marco conceptual se accede al conocimiento científico. Las normas son reglas a las que deben ceñirse las conductas, tareas y actividades del ser humano, y deben elaborarse con base en los avances del conocimiento científico. Por lo tanto, las normas internacionales de contabilidad deben ajustarse al Marco conceptual y no al revés.

La historia de la humanidad está plagada de ejemplos de lo contraproducente que resulta el hecho de que las normas que regulan las relaciones de los seres humanos se anquilosen y rezaguen con respecto al avance del conocimiento científico. Las normas no pueden detener los avances de la ciencia; en cambio, la ciencia sí debe jalonar la actualización de las normas que nos rigen. 
El Marco conceptual, por su propia naturaleza, debe ser de aplicación general, debe ser la vía de acceso a los avances del conocimiento contable y guiar la elaboración de las normas internacionales de contabilidad.

Segunda deficiencia: "Aunque una parte importante de su contenido presenta una proposición que trata de exponer de manera precisa y única la comprensión de un término, en este caso contable, logrando tipificaciones y diferenciaciones que en la mayoría de los casos se pueden considerar teoréticas, introduce algunos elementos sustantivos en la discusión teórica contable, con un tratamiento bastante limitado y ambiguo, que aunque deja percibir una problemática por resolver, no deja sentadas las definiciones conceptuales, típicas de un marco conceptual".

Las definiciones conceptuales que realiza el Marco conceptual en su mayoría se circunscriben a lo que se entiende como tal. Estas definiciones permiten llegar al conocimiento científico. Aristóteles ${ }^{6}$ comienza el libro I de la Metafísica diciendo: "Todos los hombres tienen por naturaleza el deseo de saber". El deseo de saber hace avanzar el conocimiento científico. En el libro II de la misma obra, titulado "Los últimos analíticos", Aristóteles establece que una definición debe incluir el género y la diferencia específica, la clase a la que pertenece el objeto nombrado por el término definido, y las características que lo diferencian de esa clase, es decir sus especificidades.

Las definiciones planteadas alcanzan el rango de teoréticas, porque una definición teorética da el significado de una palabra en los términos de las teorías de una determinada disciplina, asume el conocimiento y la aceptación de la teoría de la que depende, y es común en contextos científicos, donde las teorías tienden a estar más precisamente definidas y los resultados son más ampliamente aceptados como correctos.

Sin embargo, muchos de los términos y conceptos definidos en el Marco conceptual, aunque logran clasificarse como teoréticos, tienen un aporte muy limitado; en su mayoría alcanzan una generalización sobre las definiciones realizadas en libros de texto y normas anteriores, pero no hacen ningún aporte nuevo mediante definiciones conceptuales que hagan avanzar la disciplina contable. Por ejemplo, las definiciones de: estados financieros, base de acumulación o devengo contable (causación), negocio en marcha, importancia relativa, ingresos, gastos, y cualidades de la información financiera: comprensibilidad, relevancia, fiabilidad y comparabilidad. 
Otras nuevas definiciones y conceptos importantes, aunque se convierten en una presentación que propicia la discusión dentro de la disciplina contable, vuelven a caer en una propuesta muy limitada y reduccionista que no realiza una definición conceptual rigurosa. Este es el caso de:

1) El concepto de probabilidad hace referencia al grado de incertidumbre con que los beneficios económicos futuros llegarán o saldrán de la entidad. Contrariamente a este criterio de incertidumbre como sinónimo de riesgo, la noción de incertidumbre de Knight (1921), coincidente con la versión austriaca, se relaciona directamente con los beneficios de los negocios. Riesgo es distinto de incertidumbre: cuando se pueden asignar probabilidades a los resultados de distintos eventos, ya no existe incertidumbre y aparece la noción de riesgo.

Por su parte, Mises (1949,p. 130) contribuye a la discusión sobre probabilidades. Argumenta que hay dos especies de probabilidad totalmente distintas: la que se puede denominar de clase (o de frecuencia) y la de caso (es decir, la que se da en la comprensión típica de las ciencias de la acción humana). El campo en que rige la primera es el de las ciencias naturales, dominado enteramente por la causalidad; la segunda aparece en el terreno de la acción humana, plenamente regulado por la teleología.

La distinción de Knight, que relaciona incertidumbre con el beneficio económico de los negocios, así como la diferenciación de dos especies de probabilidad de Mises, entre otras, deben tenerse en cuenta en el Marco conceptual, a efectos de propiciar una discusión que permita establecer qué tan viable es el manejo de probabilidades en todos los renglones de un negocio. A pesar de las limitaciones, bienvenida la discusión.

2) También las dimensiones predictiva y confirmativa de la información que se plantean en el concepto de relevancia son muy limitadas. El documento propone que estas dimensiones están interrelacionadas. Para explicarlo utiliza un ejemplo:

La información acerca del nivel actual y la estructura de los activos poseídos tiene valor para los usuarios cuando se esfuerzan al predecir la capacidad de la entidad para aprovechar sus oportunidades y su capacidad para reaccionar ante situaciones adversas. La misma información juega un papel confirmatorio respecto a predicciones anteriores sobre, por ejemplo, la manera en que la entidad se estructuraría o sobre el resultado de las actividades planificadas. 
Por el ejemplo puede deducirse que la introducción del cálculo de probabilidades se abandona. Ya no se habla de probabilidades de ganancia o de pérdidas, sino del esfuerzo para predecir y la posibilidad de confirmación de predicciones anteriores. Nuevamente aparece la ambigüedad. Nuevamente el Marco conceptual abandona su razón de ser.

3) Otra definición contradictoria es la fiabilidad entendida como libre de error material y de sesgo o prejuicio. Argumenta el documento que los usuarios pueden confiar en que es la imagen fiel de lo que pretende representar, o de lo que puede esperarse razonablemente que represente. Si es la imagen fiel, cómo introducir los conceptos nuevos de incertidumbre, probabilidad, diferentes métodos de valoración, etc.

Lo anterior se reafirma cuando más adelante se argumenta que

La mayor parte de la información financiera está sujeta a algún riesgo de no ser una representación fiel de lo que pretende representar. Esto no se debe al sesgo, sino más bien a las dificultades inherentes a la identificación de las transacciones y demás sucesos que deben medirse o al diseño y aplicación de las técnicas de medición y presentación que pueden transmitir los mensajes que se correspondan con esas transacciones y sucesos.

4) Finalmente, se emplean términos sin que se defina de manera adecuada su significado y el contexto en que se aplican. Este es el caso de la "Correlación entre activos y pasivos". Más adelante se vuelve a utilizar la palabra "correlación", cuando se afirma que "Los gastos se reconocen en el estado de resultados sobre la base de una asociación directa entre los costos incurridos y la obtención de partidas específicas de ingresos. Este proceso, al que se denomina comúnmente correlación de gastos con ingresos [...]".Y finaliza asegurando que "No obstante, la aplicación del proceso de correlación, bajo este Marco conceptual, no permite el reconocimiento de partidas, en el balance, que no cumplan la definición de activo o de pasivo".

No es fácil establecer si se trata de una correlación estadística, o qué otro tipo de correlación pueda calcularse entre activos y pasivos, o entre ingresos y gastos. Definiciones bastante imprecisas por tratarse de un marco conceptual, que termina alejado del objetivo y de su propia razón de ser. 


\section{Referencias}

Borsotti, C. (1990). Apuntes sobre los conceptos científicos y su construcción, borrador para discusión. Universidad Nacional de Luján, Departamento de Educación, Área Metodología de la Investigación.

Bréhier, E. (1988). Historia de la filosofía (2 vols.). Traducción de Juan Antonio Pérez Millán y $\mathrm{M}^{\mathrm{a}}$ Dolores Morán. Madrid: Editorial Tecnos.

Edvinsson, L. \& Malone, M. (1998). El capital intelectual. Editorial Norma.

Hayek, F.A. (1972). Individualism and Economic Order. Chicago, IL.: Gateway edn. Henry Regnery.

Hicks, J.R. (1939). Valor y capital. Quinta reimpresión, 1977. Fondo de Cultura Económica.

Mises, L. von. (1949). La acción humana. Madrid: Unión Editorial, 1995.

Oakeshott,M.(1991).Rationalism in Politics and Other Essays. Indianapolis: Liberty Press.

Polanyi, M. (1959). The Study of Man. Chicago, IL.: University of Chicago Press.

Tobin, J. (1969, sept.). A General Equilibrium Approach To Monetary Theory. En Journal of Money, Credit and Banking, vol. 1, No. 1, pp. 15-29. 